\title{
A Method for the Suppression of Fluctuations in the Neutral-Point Potential of a Three-Level NPC Inverter with a Capacitor-Voltage Loop
}

\author{
Yun Zhang, Member, IEEE, Jing Li, Xinmin Li, Yanfei Cao, Mark Sumner, Senior Member, IEEE, and \\ Changliang Xia, Senior Member, IEEE
}

\begin{abstract}
This paper investigates the problem of fluctuation of the neutral-point potential (NPP) in a three-level NPC inverter with a capacitor-voltage loop. The phase pulse width duty cycle disturbance PWM method is proposed to suppress the NPP fluctuation efficiently. Based on the basic carrier-based Phase Disposition (PD) PWM method, the average pulse neutral-point current model is established. Then the frequency, amplitude and equivalent initial phase of the NPP fluctuation are analyzed based on the current model. According to the alternating error of the DC-link capacitor voltages, a capacitor-voltage loop with a quasi PR (proportional resonant) controller is presented. The control variable, which varies with the modulation index, phase current, load power factor, etc, can be obtained from the quasi PR controller. Finally, an experimental three-level NPC inverter is described and the validity and feasibility of the proposed method are verified by experimental results.
\end{abstract}

Keywords: Three-level NPC inverter, neutral-point potential, capacitor-voltage loop, fluctuation-suppression.

\section{INTRODUCTION}

In order to step down $\mathrm{d} v / \mathrm{d} t$, improve the harmonic performance of the output voltage and current, and also reduce the voltage stress of power switches of inverters used in medium-voltage and high power applications, the three-level neutral-point clamped (NPC) inverter was proposed [1]. However a particular problem with the NPC compared to traditional two-level inverters, is the fluctuation of the capacitor midpoint voltage or neutral-point potential (NPP) due to influences from the modulation index and the load power factor

Manuscript received September 2, 2015. Accepted for publication February 18, 2016. This work was supported in part by the National Natural Science Foundation of China under Grants 51577130, and 51207104, and in part by the Research Program of Application Foundation and Advanced Technology of Tianjin China under Grant 15JCQNJC03900.

Yun Zhang, Xinmin Li, and Yanfei Cao are with the School of Electrical Engineering and Automation, Tianjin University, Tianjin 300072, China (fax: +86-022-27402325; e-mail: zhangy@tju.edu.cn, lixinmin@tju.edu.cn, and caoyanfei@tju.edu.cn).

Jing $\mathrm{Li}$ is with the Department of Electrical and Electronic Engineering, University of Nottingham, Ningbo, China (email: jing.li@ nottingham.edu.cn).

Mark Sumner is with the Department of Electrical and Electronic Engineering, University of Nottingham, UK (email: mark.sumner@nottingham.ac.uk).

Changliang Xia is with the School of Electrical Engineering and Automation, Tianjin University, and also with the Tianjin Key Laboratory of Advanced Technology of Electrical Engineering and Energy, Tianjin Polytechnic University, China (e-mail: motor@ tju.edu.cn).
[2]. Capacitor voltage imbalance may cause failure of the power switches and the series connected DC-link capacitors. In addition low-order harmonics will be created in the output voltage and current, due to low-frequency ripple of the NPP [3]. Therefore, a great deal of research on the NPP fluctuation problem of three-level NPC inverters has been carried out [4]. In medium-voltage induction motor drive systems, the three-level NPC inverter can eliminate NPP fluctuation by using two isolated voltage sources instead of capacitors in series $[5,6]$. In addition, the NPP fluctuation could also be removed through using a front-end converter such as a three-level boost converter or a back to back topology $[7,8]$. These additional converters can be used to inject or extract current into or from the neutral point if necessary [9]. However, with the additional hardware circuits, the volume and cost of the system are increased while the efficiency and the reliability of the system are reduced. Therefore, improving the modulation performance is a better way to restrain the NPP fluctuation as this removes the need for extra converters [10-13].

There are two main modulation strategies for the three-level NPC inverter, namely space vector pulse width modulation (SVPWM) and carrier-based PWM [14, 15]. The nearest three vectors (NTV) modulation is one of the common SVPWM methods [16]. It conforms to the principle in which the reference vector is synthesized by the nearest three vectors and can prevent overlapping of the level layers in the line to line voltages (analyzed in [17]), as well as reducing the total harmonic distortion (THD). However, the essence of the NTV modulation method is that a third-order harmonic is injected into the three-phase sinusoidal modulation waves, and its fluctuation-suppression for NPP is limited (this is analyzed in detail in Section III. A). Therefore, the nearest three virtual vectors $\left(\mathrm{NTV}^{2}\right)$ method was proposed to enhance the capability for fluctuation-suppression [18, 19]. Compared to the NTV method, the $\mathrm{NTV}^{2}$ method goes against the principle of the synthesized reference vector, and produces overlapping of level layers and as much as $25 \%$ more switching actions in every fundamental period. Naturally, the hybridization of the NTV and the $\mathrm{NTV}^{2}$ methods is a tradeoff between the fluctuation-suppression and the fundamental switching number. The proportional index $p_{\mathrm{m}}$, such as $p_{\mathrm{m}}=0.5$, was used to determine the extent of the alternate action in a fundamental period [20], but the overlapping of level layers still exists in this proportional hybrid SVPWM strategy in line to line voltages. 
The three-level NPC inverter is suitable for electric power plants, municipal water supply systems, etc. It is mainly used in medium-voltage and high power variable voltage variable frequency (VVVF) induction motor drive systems [21, 22]. Therefore, according to the designed voltage-frequency curve, the required speed adjustment of the fan or pump results in the induction motor's variable voltage and power factor, as well as its phase currents [23]. However, the combinations of the variable voltage, power factor and phase current due to the changed speed of the induction motor may affect the neutral-point current (namely the neutral-point potential) of the NPC inverter. A basic carrier-based PD PWM method with a capacitor-voltage loop is proposed in this paper, in order to solve the problem of the NPP fluctuation, and avoid the disadvantages (more switching actions and overlapping levels of the line to line voltages) of the $\mathrm{NTV}^{2}$, the hybridization of the NTV and the NTV ${ }^{2}$ methods mentioned above. This proposed method can also reduce the impact of the practical imbalance capacitance of the series connected DC-link capacitors.

Moreover, the VVVF control is adopted for the inverter to supply a three-phase $R L$ load (for the convenience of exactly obtaining the combinations of the variable voltage, power factor and phase current) in this paper. It thus begins with the theoretical analysis of the NPP fluctuation frequency, amplitude, and equivalent initial phase, based on the basic carrier-based PD PWM method. Then, the NPP fluctuation method with injected $3^{\text {rd }}$ harmonic PWM is introduced with its limited ability to suppress the NPP fluctuation, due to the constant initial phase of the injected $3^{\text {rd }}$ harmonic component. The capacitor-voltage loop with the quasi PR controller that is presented here can create a variable zero sequence component, i.e. a variable equivalent initial phase, according to the load power factor. Furthermore, the proposed phase pulse width duty cycle disturbance PWM method changes the modulation waves in the presence of NPP, resulting in an efficient suppression of NPP fluctuation, as well as less switching actions, non-overlapping levels of the line to line voltages, and tolerance to the imbalance capacitance of the series connected DC-link capacitors.

\section{BASIC ANALYSIS OF NPP FLUCTUATION \\ A. Frequency of NPP Fluctuation}

The topology of the three-level NPC inverter is shown in Fig. 1. The capacitance of the DC-link capacitors $\mathrm{C}_{\text {cap1 }}, \mathrm{C}_{\text {cap2 }}$ are equal $\left(C_{1}=C_{2}\right)$, and the DC-link voltage $U_{\mathrm{dc}}$ is divided into $U_{\text {cap } 1}=U_{\text {cap2 }}=U_{\mathrm{dc}} / 2$. Point "o" is the neutral point. The basic carrier-based PD PWM method is adopted to control the power switches $\mathrm{Q}_{\mathrm{x} 1} \sim \mathrm{Q}_{\mathrm{x} 4}$, where $\mathrm{x}$ stands for Phases $\mathrm{a}, \mathrm{b}$, and $\mathrm{c}$.

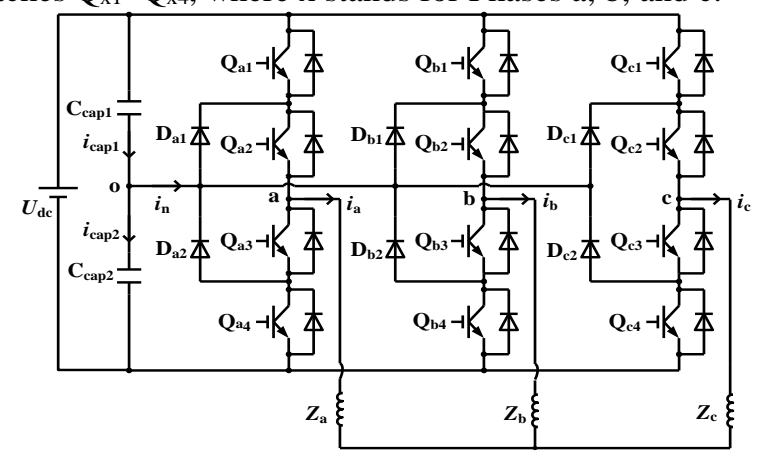

Fig. 1. Topology of three-level NPC inverter.
The neutral-point potential $U_{\mathrm{o}}$ can be regarded as the voltage across $\mathrm{C}_{\text {cap2 }}$ as follows

$$
U_{\mathrm{o}}=U_{\text {cap2 }}
$$

Given that medium or small vectors cause corresponding NP current during the action time, so $\mathrm{C}_{\text {cap } 1}$ is charged or discharged, and $\mathrm{C}_{\text {cap2 }}$ is discharged or charged, under the condition of $U_{\text {cap1 }}+$ $U_{\text {cap2 }} \equiv U_{\text {dc }}$. The voltage ripple of $\mathrm{C}_{\text {cap2 }}$ is determined by $i_{\mathrm{n}}$ and the action time $\mathrm{d} t(\mathrm{~d} t<<T, T$ is the fundamental period), by virtue of the corresponding vector. From Fig. 1, it can be seen that the voltage ripple $\Delta u_{2}$ of $\mathrm{C}_{\text {cap2 }}$ is the difference between $U_{\text {cap2 }}$ and $U_{\mathrm{dc}} / 2$, namely the NPP fluctuation $\Delta u_{\mathrm{o}}$ is written as

$$
\Delta u_{\mathrm{o}}=\Delta u_{2}=U_{\text {cap2 }}-U_{\mathrm{dc}} / 2=\frac{1}{C_{2}} \int_{0}^{t_{\text {cep }}} i_{\text {cap } 2} \mathrm{~d} t
$$

where $i_{\text {cap2 } 2}$ is the instantaneous current flowing into or out of

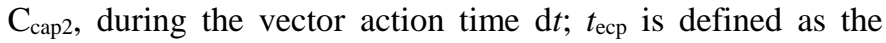
effective charging or discharging time when $U_{\text {cap2 }}$ goes from $U_{\mathrm{dc}} / 2$ to the peak value. According to Fig. 1, when medium or small vectors act, the current equation concerning the neutral point " $\mathrm{o}$ " can be obtained as follows

$$
i_{\text {cap } 1}=i_{\text {cap } 2}+i_{\mathrm{n}}
$$

The charging or discharging state of $\mathrm{C}_{\text {cap2 }}$ is shown in Fig. 2. This results from the NP current $i_{\mathrm{n}}$ when using the basic carrier-based PD PWM method over a fundamental period. In fact, $i_{\mathrm{n}}$ is the pulsed NP current, as shown in Fig. 2(e). Its pulse amplitude corresponds to the instantaneous phase current. In addition, the three-phase sinusoidal modulation waves are divided into six regions I VI, as depicted in Fig. 2(a). The phase pulse width duty cycle is defined as the ratio of the positive or negative phase voltage pulse width to the carrier period, as denoted in Fig. 2(b d). The phase pulse width duty cycle $d_{\mathrm{x}}$ can also be defined as the ratio of the modulation wave instantaneous value $(-1 \sim 1)$ to the corresponding carrier amplitude (1 or -1$)$, i.e. it can be expressed as

$$
d_{\mathrm{x}}=\left|u_{\text {ref_x }}\right|
$$

where $d_{\mathrm{x}}=0 \sim 1$. According to Fig. $2(\mathrm{e})$, it is noted that the pulse NP current $i_{\mathrm{n}}$ varies with both the duty cycles and the phase currents. As a result, the average current flowing into the neutral point can be described as

$$
\bar{i}_{\mathrm{n}}=-\left(d_{\mathrm{a}} \times i_{\mathrm{a}}+d_{\mathrm{b}} \times i_{\mathrm{b}}+d_{\mathrm{c}} \times i_{\mathrm{c}}\right)
$$

where $\bar{i}_{\mathrm{n}}$ is the average pulse NP current; $d_{\mathrm{a}}, d_{\mathrm{b}}$ and $d_{\mathrm{c}}$ are phase pulse width duty cycles; $i_{\mathrm{a}}, i_{\mathrm{b}}$, and $i_{\mathrm{c}}$ are three-phase instantaneous currents.

The three-phase sinusoidal modulation waves are defined as

$$
\left\{\begin{array}{l}
u_{\text {ref_a }}=m \sin \omega t \\
u_{\text {ref_b }}=m \sin (\omega t-2 \pi / 3) \\
u_{\text {ref_c }}=m \sin (\omega t-4 \pi / 3)
\end{array}\right.
$$

where $m$ is the modulation index, and $0 \leq m \leq 1 ; \omega$ is the fundamental angular frequency. When the carrier frequency is much greater than the fundamental frequency and the three-level NPC inverter supplies a three-phase symmetrical $R L$ load, the phase currents can be considered as sinusoidal 


$$
\left\{\begin{array}{l}
i_{\mathrm{a}}=I \sin (\omega t-\varphi) \\
i_{\mathrm{b}}=I \sin (\omega t-2 \pi / 3-\varphi) \\
i_{\mathrm{c}}=I \sin (\omega t-4 \pi / 3-\varphi)
\end{array}\right.
$$

where " $I$ " is the phase current amplitude, and $\varphi$ is the load power factor angle. Therefore, in terms of (4), each phase pulse width duty cycle in Region I $(\omega t \in[0, \pi / 3))$ as shown in Fig. 2(a), can be written as follows

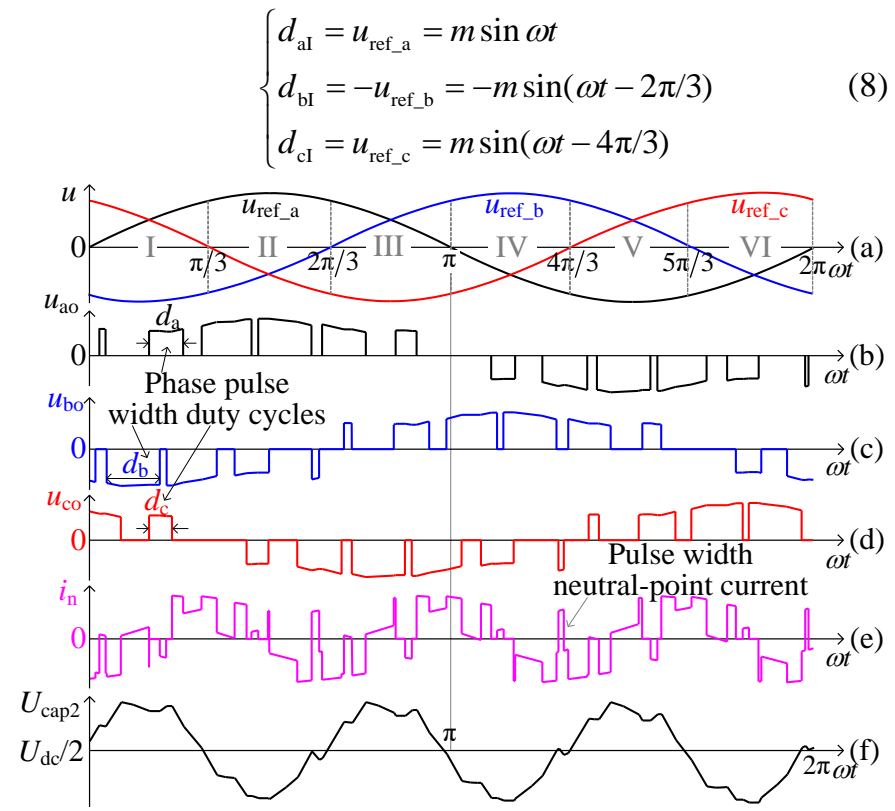

Fig. 2 Charging or discharging state of $\mathrm{C}_{\text {cap } 2}$ by NP current $i_{\mathrm{n}}$ under basic carrier-based PD PWM method during a fundamental period.

According to (5), (7) and (8), the average pulse NP current

$\bar{i}_{\mathrm{nI}}$ in Region I can be obtained as

$$
\begin{aligned}
\bar{i}_{\mathrm{nI}} & =-m \sin (\omega t) \times I \sin (\omega t-\varphi) \\
& -[-m \sin (\omega t-2 \pi / 3)] \times I \sin (\omega t-2 \pi / 3-\varphi) \\
& -m \sin (\omega t-4 \pi / 3) \times I \sin (\omega t-4 \pi / 3-\varphi) \\
& =-0.5 m I[\cos \varphi+2 \cos (2 \omega t-4 \pi / 3-\varphi)]
\end{aligned}
$$

Likewise, the average pulse NP current in the other five regions can also be deduced, and the current model $i_{\text {n }}$ can be established in a fundamental period $\omega t \in[0,2 \pi)$ as follows

$$
\bar{i}_{\mathrm{n}}=\left\{\begin{array}{l}
0.5 m I[-\cos \varphi-2 \cos (2 \omega t-4 \pi / 3-\varphi)], \omega t \in[0, \pi / 3) \\
0.5 m I[\cos \varphi+2 \cos (2 \omega t-\varphi)], \omega t \in[\pi / 3,2 \pi / 3) \\
0.5 m I[-\cos \varphi-2 \cos (2 \omega t-2 \pi / 3-\varphi)], \omega t \in[2 \pi / 3, \pi) \\
0.5 m I[\cos \varphi+2 \cos (2 \omega t-4 \pi / 3-\varphi)], \omega t \in[\pi, 4 \pi / 3) \\
0.5 m I[-\cos \varphi-2 \cos (2 \omega t-\varphi)], \omega t \in[4 \pi / 3,5 \pi / 3) \\
0.5 m I[\cos \varphi+2 \cos (2 \omega t-2 \pi / 3-\varphi)], \omega t \in[5 \pi / 3,2 \pi)
\end{array}\right.
$$

From (10), each of the expressions shows the frequency of " $\bar{i}_{\mathrm{n}}$ " is twice the fundamental frequency during $\omega t \in[0,2 \pi)$. In

fact, the actual $i_{\mathrm{n}}$ is synthesized by the corresponding part of every expression in terms of the exact range of $\omega t$ shown in (10). As a result, there exists the unique time $t_{\mathrm{I}}$ when $\bar{i}_{\mathrm{n}}=0$ during $\omega t \in[0, \pi / 3)$. Thus $\omega t_{\mathrm{I}}$ can be written as

$$
\omega t_{\mathrm{I}}=0.5 \times \bmod \{[4 \pi / 3+\varphi+\arccos (-0.5 \cos \varphi)], 2 \pi\}
$$

where "mod" is the remainder function. Similarly, the other five expressions $\omega t_{\mathrm{II}} \sim \omega t_{\mathrm{VI}}$ can also be obtained in the other five regions. Consequently, there are six zero crossing points of $i_{\mathrm{n}}$ in a fundamental period according to (10) i.e. $\left(\omega t_{\mathrm{I}}, 0\right),\left(\omega t_{\mathrm{II}}, 0\right)$, $\left(\omega t_{\mathrm{III}}, 0\right),\left(\omega t_{\mathrm{IV}}, 0\right),\left(\omega t_{\mathrm{V}}, 0\right)$ and $\left(\omega t_{\mathrm{VI}}, 0\right)$ and the frequency of $\bar{i}_{\mathrm{n}}$ is three times the fundamental one. The DC-link capacitor voltages also fluctuate at three times the fundamental frequency. The simulation results of the neutral-point current and the fluctuating state of the capacitor voltage $U_{\text {cap2 }}$ are shown in Fig. 3 , from a Matlab/Simulink simulation with the simulation condition of $m=1$, and $\cos \varphi=0.886$.

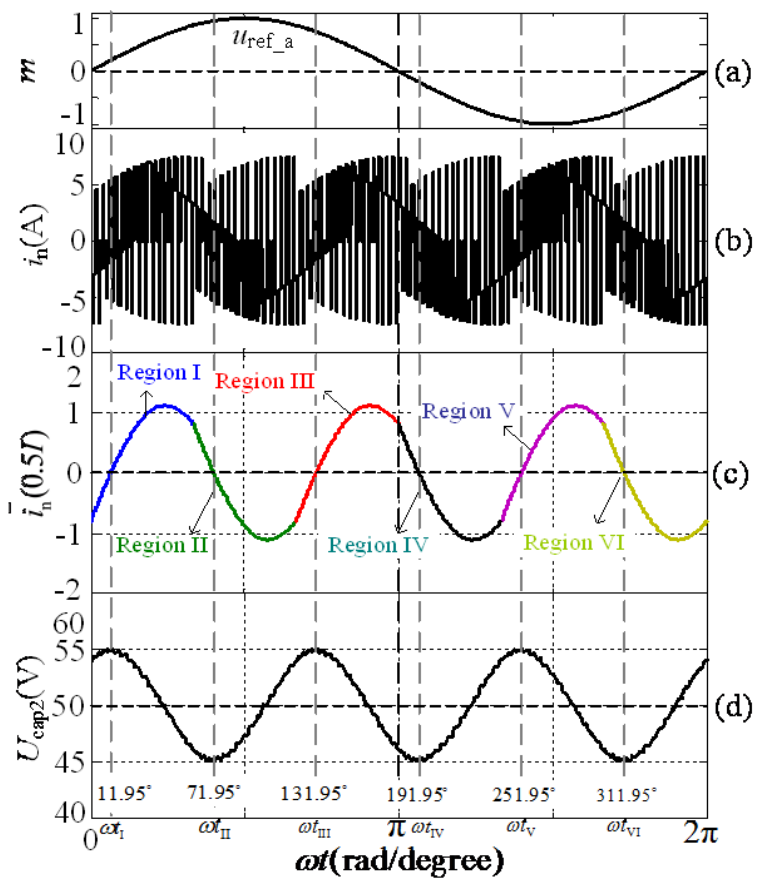

Fig. 3 Simulation results of neutral-point current and fluctuating state of capacitor voltage from Matlab/Simulink (simulation parameters: $m=1$, fundamental frequency $f=50 \mathrm{~Hz}$, switching frequency $f_{\mathrm{c}}=4.67 \mathrm{kHz}$,

$C_{1}=C_{2}=470 \mu \mathrm{F}, U_{\mathrm{dc}}=100 \mathrm{~V}$, load $R=6 \Omega, L=10 \mathrm{mH}$, and $\cos \varphi=0.886$ ).

(a) Sinusoidal modulation wave. (b) Pulse NP current. (c) Average pulse NP current. (d) Fluctuating voltage of $\mathrm{C}_{\text {cap2 }}$.

The "Phase-a" sinusoidal modulation wave $u_{\text {ref_a }}$ is shown in Fig. 3(a), and the pulse NP current $i_{\mathrm{n}}$ is depictured in Fig. 3(b). In terms of (10), the average pulse NP current is shown in Fig. 3(c), and its amplitude is determined by $m, I$, and $\varphi$. Its frequency is three times the fundamental as analyzed above. Moreover, the charging and discharging characteristics for DC-link capacitors can be explained more clearly by $\bar{i}_{\mathrm{n}}$ : In Region I, $\bar{i}_{\mathrm{n}}$ (blue curve) is from negative to positive with the zero crossing point $\left(\omega t_{\mathrm{I}}, 0\right)$, where $\omega t_{\mathrm{I}}=11.95^{\circ}$ is calculated by (11) with $\cos \varphi=0.886$; when $\bar{i}_{\mathrm{n}}<0, \mathrm{C}_{\text {cap } 1}$ is being discharged, and $\mathrm{C}_{\text {cap2 }}$ is being charged; when $\bar{i}_{\mathrm{n}}=0, \mathrm{C}_{\text {cap1 }}$ is discharged down to the minimum voltage, and $\mathrm{C}_{\text {cap2 }}$ is charged 
up to the maximum; when $\bar{i}_{\mathrm{n}}>0, \mathrm{C}_{\text {cap } 1}$ is being charged, and $\mathrm{C}_{\text {cap2 }}$ is being discharged. When $i_{\mathrm{n}}$ (green curve) is in Region II, another zero crossing point occurs $\left(\omega t_{\mathrm{II}}, 0\right)$ at $\omega t_{\mathrm{II}}=71.95^{\circ}$. At this time, $\mathrm{C}_{\mathrm{cap} 1}$ is charged up to the maximum voltage, and $\mathrm{C}_{\text {cap2 }}$ is discharged down to the minimum as shown in Fig. 3(d). The fluctuation frequency of $U_{\text {cap2 }}$ is three times the fundamental as shown above.

\section{B. Amplitude of NPP Fluctuation}

According to (2), (10) and the conclusions for the NPP fluctuation frequency analyzed above, then by examining $U_{\mathrm{dc}} / 2$, the neutral-point potential $U_{\mathrm{o}}$ is at its maximum or minimum voltage during $T / 12$. Therefore, the amplitude of the NPP fluctuation is directly determined by $i_{\text {cap } 1}, i_{\text {cap2 }}, C_{1}, C_{2}, t_{\text {ecp }}$ in $T / 12, m, I$, and $\varphi$. It is clear that the NPP fluctuation is inversely proportional to $C_{1}$ and $C_{2}$.

It should be noted that the charging or discharging states of $\mathrm{C}_{\text {cap } 1}$ and $\mathrm{C}_{\text {cap2 }}$ are opposite from each other, with the condition that $U_{\text {cap } 1}+U_{\text {cap } 2} \equiv U_{\mathrm{dc}}$. When $C_{1}, C_{2}, \omega, m$, and $\varphi$ remain unchanged, $i_{\text {cap } 1}$ and $i_{\text {cap2 }}$ are proportional to $i_{\mathrm{n}}$ according to (3). $i_{\text {cap2 }}$ is proportional to $I$, as well as the amplitude of the NPP fluctuation by virtue of (2); when the fundamental angular frequency $\omega$ is decreased from $\omega_{1}$ to $\omega_{2}$, the period of $i_{\mathrm{n}}$ increases, leading to a longer distance between adjacent zero crossing points. Therefore the effective time $t_{\mathrm{ecp}}$, which is the equivalent time for charging or discharging capacitors from $U_{\mathrm{dc}} / 2$ to the maximum or minimum voltage, increases proportionally from $t_{\mathrm{ecp} 1}$ to $t_{\mathrm{ecp} 1} \times \omega_{1} / \omega_{2} \cdot t_{\mathrm{ecp} 1}$ is the equivalent charging or discharging time when the fundamental angular frequency is $\omega_{1}$. In addition, the amplitude of phase currents remains unchanged. Consequently the amplitude of the NPP fluctuation is $\omega_{1} / \omega_{2}$ times that of the original one, as shown in Fig. 4, i.e. it is inversely proportional to the fundamental frequency.

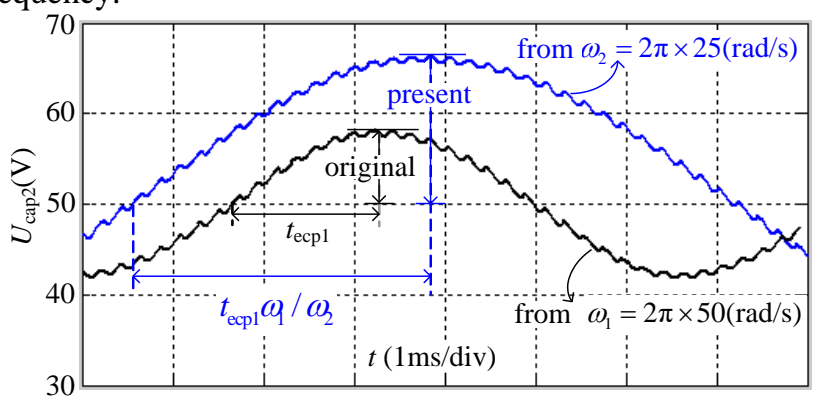

Fig. 4 Comparative amplitudes of NPP fluctuation between conditions of $\omega_{1}=2 \pi \times 50(\mathrm{rad} / \mathrm{s}), L=10 \mathrm{mH}$ and $\omega_{2}=2 \pi \times 25(\mathrm{rad} / \mathrm{s}), L=20 \mathrm{mH}(R=6 \Omega)$.

In order to clarify the impacts of the modulation index and load power factor on the NPP fluctuation, an analysis of the three-level NPC inverter supplying a three-phase symmetrical $R L$ load is made here. It is assumed that the load power factor is constant $\cos \varphi=0.866$ ( $R=5.89 \Omega, L=10.8 \mathrm{mH}$, fundamental frequency $f=50 \mathrm{~Hz}$ ), while $m$ is set to two conditions namely " 0.533 " and " 1 ". The other simulation parameters are the same with those used for Fig. 3. The comparison of fluctuation amplitudes of the capacitor voltages with different modulation indexes is shown in Fig. 5. The amplitudes of $i_{\mathrm{n}}$ in Fig. $5(\mathrm{a}, \mathrm{b})$ are $0.6 \times 0.5 I$ and $1.13 \times 0.5 I$, respectively; and the fluctuation amplitude $\Delta U_{2}$ of $U_{\text {cap2 }}$ is $1.4 \mathrm{~V}$ and $5 \mathrm{~V}$. As shown in (10), with

a constant $\varphi$, the amplitude of $i_{\mathrm{n}}$ is determined by $m$ and $I$. The fluctuation amplitudes of the capacitor voltages are nearly proportional to the amplitude of $\bar{i}_{\mathrm{n}}$. For an identical $R L$ load and fundamental frequency, $I$ is proportional to $m$. Therefore $i_{\mathrm{n}}$ is proportional to $m I$, i.e. the fluctuation amplitude $\Delta U_{2}$ of $U_{\text {cap2 }}$ is proportional to $m^{2}$.

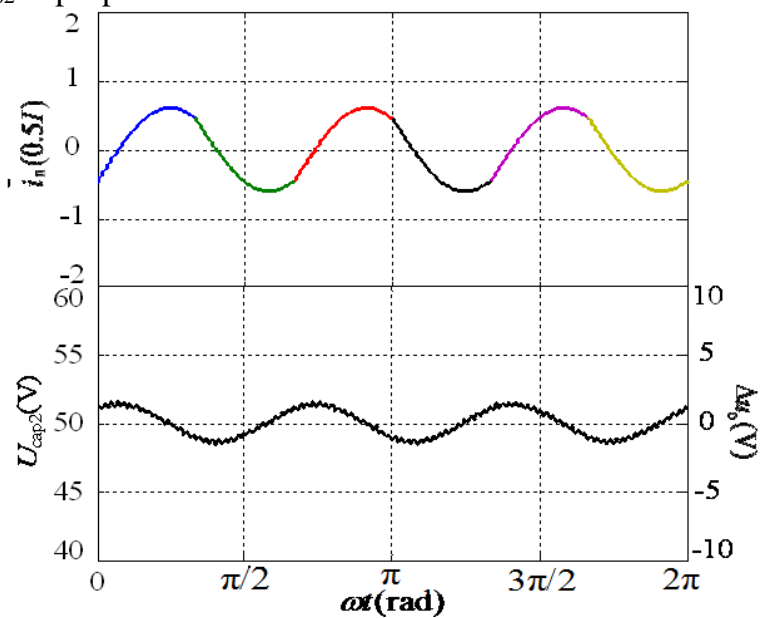

(a) $m=0.533$

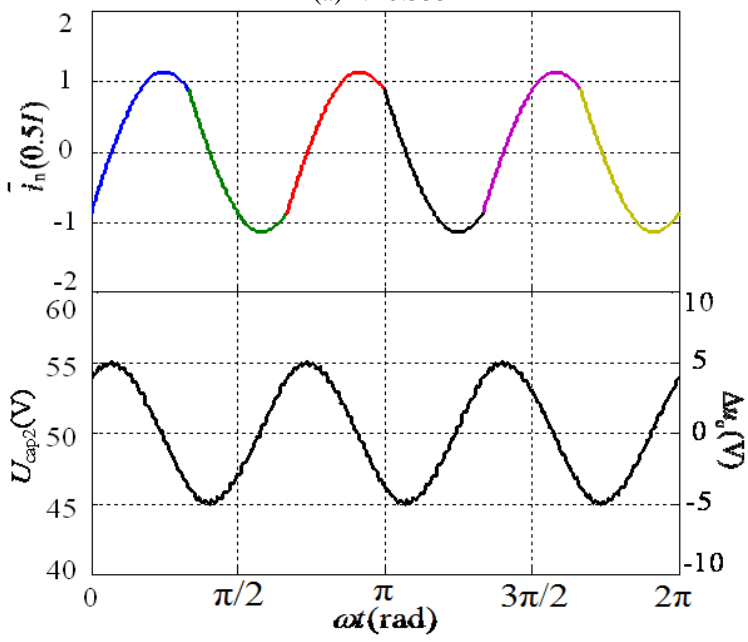

(b) $m=1$

Fig. 5 Comparison of fluctuation amplitudes of capacitor voltages with different modulation indexes $(R=5.89 \Omega, L=10.8 \mathrm{mH}, f=50 \mathrm{~Hz}$, and $\cos \varphi=0.866)$.

Regarding the impact of the load power factor, it is assumed that $m=1$, the phase impedance $Z_{\text {phase }}=6.8 \Omega$, and $f=50 \mathrm{~Hz}$. With the same phase impedance, different load power factors can be obtained by adjusting the phase resistors and inductors, e.g. $0.996\left(\varphi=5^{\circ}\right), 0.866\left(\varphi=30^{\circ}\right), 0.5\left(\varphi=60^{\circ}\right)$ and 0.087 $\left(\varphi=85^{\circ}\right.$ ). The other simulation parameters remain the same as those in Fig. 3. The comparison of the fluctuation amplitudes of the capacitor voltages under different load power factors is shown in Fig. 6. 


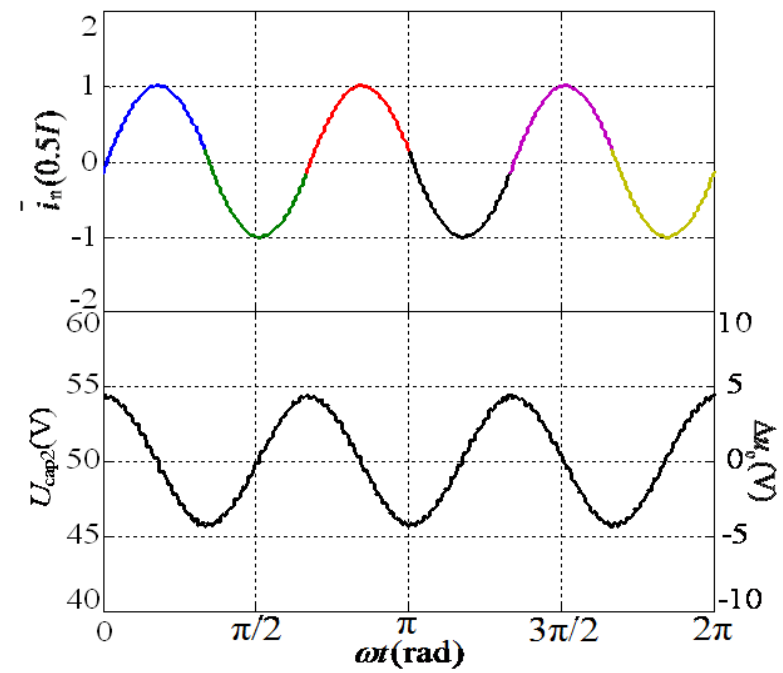

(a) $R=6.77 \Omega, L=1.89 \mathrm{mH}, \varphi=5^{\circ}$

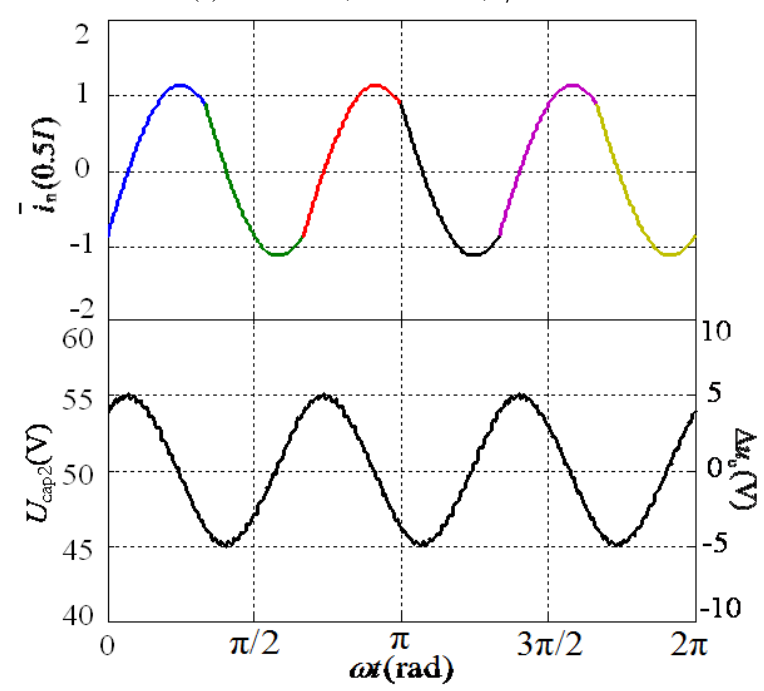

(b) $R=5.89 \Omega, L=10.8 \mathrm{mH}, \varphi=30^{\circ}$

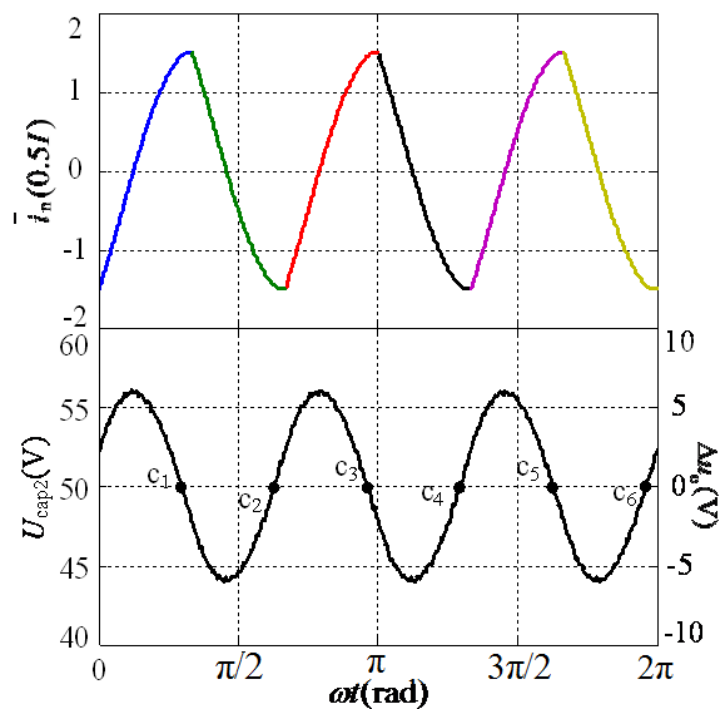

(c) $R=3.4 \Omega, L=18.75 \mathrm{mH}, \varphi=60^{\circ}$

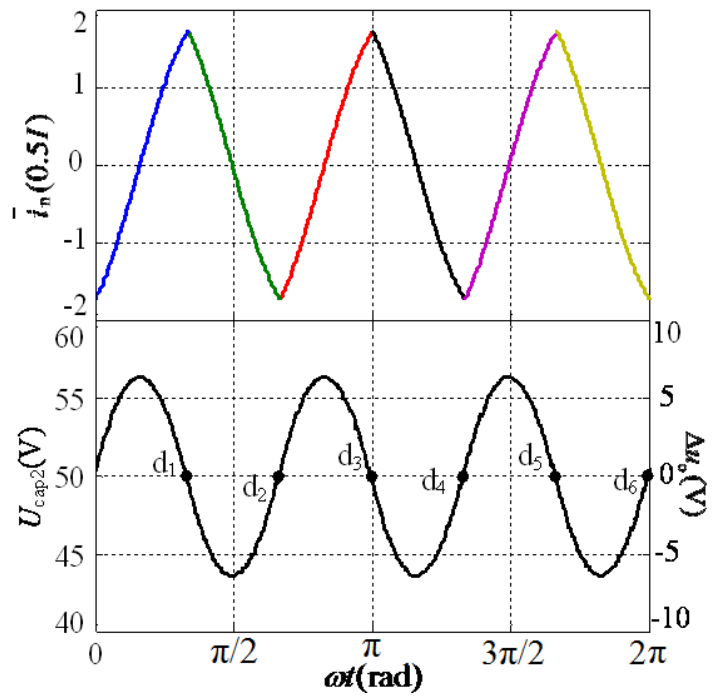

(d) $R=0.59 \Omega, L=21.57 \mathrm{mH}, \varphi=85^{\circ}$

Fig. 6 Comparison of fluctuation amplitudes of capacitor voltages with different load power factors $\left(m=1, Z_{\text {phase }}=6.8 \Omega\right.$, and $\left.f=50 \mathrm{~Hz}\right)$.

In terms of (11) and Fig. 3, there is a peak of $i_{\mathrm{n}}$ when the phase lags $\omega t_{\mathrm{I}}$ is $\pi / 6$. Combining this with (10), the amplitude function $\bar{I}_{\mathrm{n}}(\varphi)$ of $\bar{i}_{\mathrm{n}}$ can be obtained as follows

$$
\left\{\begin{array}{l}
\omega t_{\mathrm{I}}=0.5 \times \bmod \{[4 \pi / 3+\varphi+\arccos (-0.5 \cos \varphi)], 2 \pi\} \\
\bar{I}_{\mathrm{n}}(\varphi)=0.5 I\left\{-\cos \varphi-2 \cos \left[2\left(\omega t_{\mathrm{I}}+\pi / 6\right)-4 \pi / 3-\varphi\right]\right\}
\end{array}\right.
$$

where $\varphi \in[0, \pi / 2]$. According to (12), the relationship between $\bar{I}_{\mathrm{n}}$ and $\varphi$ is shown in Fig. 7. It should be noted that the linear relationship between $\bar{I}_{\mathrm{n}}$ and $\varphi$ is the "cb" line segment, and the nonlinear relationship takes place on the "ba" curve. Moreover, the nonlinear segment "ba" shows that the higher the load power factor is, the lower the influence on $\bar{i}_{\text {n }}$ from $\varphi$, as shown in Fig. 6. As a result, the NPP fluctuation amplitude $\Delta U_{\mathrm{o}}$ is proportional to $\bar{I}_{\mathrm{n}}=f(\varphi)$ as shown in Fig. 7 , according to the charging or discharging characteristic of $\mathrm{C}_{\text {cap } 1}$ and $\mathrm{C}_{\text {cap2 }}$ as stated above.

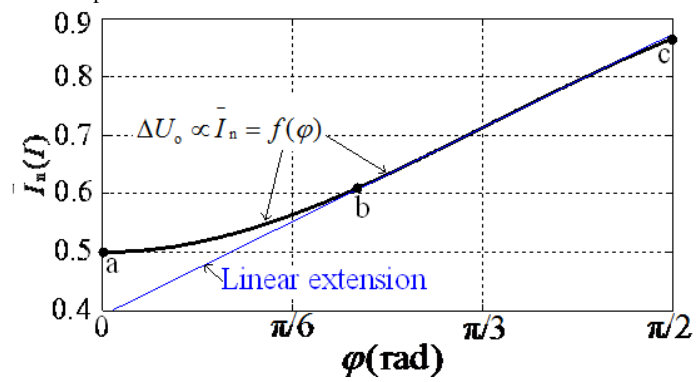

Fig. 7 Relationship between $I_{\mathrm{n}}$ and $\varphi\left(m=1\right.$, and $Z_{\text {phase }}=$ Constant $)$.

\section{Equivalent Initial Phase of NPP Fluctuation}

The NPP fluctuation $\Delta u_{\mathrm{o}}$ alternates at triple fundamental frequency and its equivalent initial phase can be considered as $3(\omega t+\theta)$, referring to the phase $\omega t$ of the sinusoidal modulation wave $u_{\text {ref_a }}=m \sin \omega t$. The phase of $\Delta u_{\mathrm{o}}$ is 
determined by the load power factor angle $\varphi$ in terms of Fig. 3 and (11), then the initial phase angle $\theta$ can be written as

$$
\begin{aligned}
& \theta=\pi / 6-\omega t_{\mathrm{I}} \\
& =\pi / 6-0.5 \times \bmod \{[4 \pi / 3+\varphi+\arccos (-0.5 \cos \varphi)], 2 \pi\}
\end{aligned}
$$

The relationship between $\theta$ and $\varphi$ is shown in Fig. 8. It is noted that $\theta$ decreases from $\pi / 6$ to zero, according to the increase of $\varphi \in[0, \pi / 2]$. As a result, six zero crossing points of $\Delta u_{\mathrm{o}}$ all cause a right shift from points $\mathrm{c}_{1} \sim \mathrm{c}_{6}$ as shown in Fig. 6(c) to the corresponding ones $d_{1} \sim d_{6}$ in Fig. $6(d)$. It reveals the substantial change of the initial phase due to the impact of the load power factor.

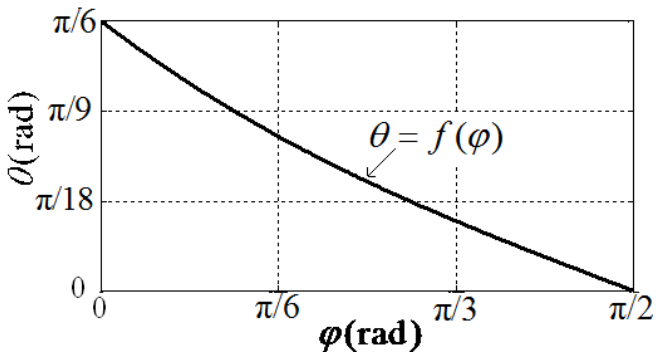

Fig. 8 Relationship between $\theta$ and $\varphi$.

\section{FluCtUATION-SUPPRESSION METHOD OF NPP}

A. NPP Fluctuation with Injected 3rd Harmonic PWM

It is known that the injected $3^{\text {rd }}$ harmonic $(m \sin 3 \omega t / 6)$ PWM method not only can improve the utilization of the DC-link voltage of the three-phase inverter, but does not change the characteristics of line to line voltages [24]. According to (5), (7), (8), and (10), the injected $3^{\text {rd }}$ harmonic can also cause a corresponding average pulse NP current $i_{\mathrm{n}}^{z}$ shown in (14)

$$
i_{\mathrm{n}}^{z}=\left\{\begin{array}{l}
m I[-\cos (4 \omega t-2 \pi / 3-\varphi)+\cos (2 \omega t+2 \pi / 3+\varphi)] / 6, \omega t \in[0, \pi / 3) \\
m I[\cos (4 \omega t-\varphi)-\cos (2 \omega t+\varphi)] / 6, \omega t \in[\pi / 3,2 \pi / 3) \\
m I[-\cos (4 \omega t-4 \pi / 3-\varphi)+\cos (2 \omega t+4 \pi / 3+\varphi)] / 6, \omega t \in[2 \pi / 3, \pi) \\
m I[\cos (4 \omega t-2 \pi / 3-\varphi)-\cos (2 \omega t+2 \pi / 3+\varphi)] / 6, \omega t \in[\pi, 4 \pi / 3) \\
m I[-\cos (4 \omega t-\varphi)+\cos (2 \omega t+\varphi)] / 6, \omega t \in[4 \pi / 3,5 \pi / 3) \\
m I[\cos (4 \omega t-4 \pi / 3-\varphi)-\cos (2 \omega t+4 \pi / 3+\varphi)] / 6, \omega t \in[5 \pi / 3,2 \pi)
\end{array}\right.
$$

As a result, the actual synthesized average pulse NP current $\overline{i_{\mathrm{n}}^{\prime}}=\overline{i_{\mathrm{n}}}+\overline{i_{\mathrm{n}}^{z}}$ can be obtained. The injected modulation wave of "Phase-a" is $u_{\text {ref_a }}^{\prime}=m \sin \omega t+(m \sin 3 \omega t) / 6$, and the injected $u_{\text {ref_b }}^{\prime}, u_{\text {ref_c }}^{\prime}$ lag $u_{\text {ref_a }}^{\prime} 120^{\circ}$ and $240^{\circ}$ respectively.

According to (10) and (14), the average pulse NP currents with injected $3^{\text {rd }}$ harmonic PWM method under the VVVF control mode are shown in Fig. 9. When $m=1, f=50 \mathrm{~Hz}$, and $\cos \varphi=0.886$, the injected average pulse NP current $\overline{i_{\mathrm{n}}^{z}}$ can offset the amplitude of the average pulse NP current $\bar{i}_{\mathrm{n}}$ by a significant amount. The amplitude of the synthesized average pulse NP current $i_{\mathrm{n}}^{\prime}$ is reduced further as shown in Fig. 9(a). Nevertheless, the injected average pulse NP current $i_{\mathrm{n}}^{z}$ cannot counteract $\overline{i_{\mathrm{n}}}$ enough when $m=0.533, f=25 \mathrm{~Hz}, \quad$ and $\cos \varphi=0.59$, the amplitude of $i_{\mathrm{n}}^{\prime}$ is virtually unchanged as shown in Fig. 9(b). Therefore, it is concluded that the injected $3^{\text {rd }}$ harmonic component, whose initial phase is constant (i.e. does not change with the modulation index, load current and power factor), cannot suppress the amplitude of the synthesized average pulse NP current $i_{\mathrm{n}}^{\prime}$ efficiently under VVVF control.

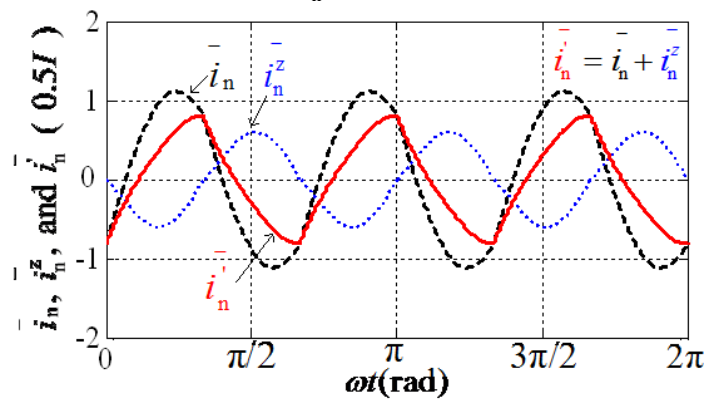

(a) $m=1, f=50 \mathrm{~Hz}, R=6 \Omega, L=10 \mathrm{mH}, \cos \varphi=0.886$

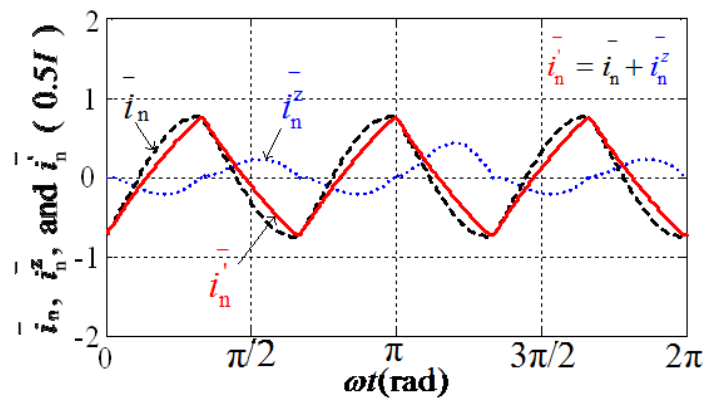

(b) $m=0.533, f=25 \mathrm{~Hz}, R=4.54 \Omega, L=40 \mathrm{mH}, \cos \varphi=0.59$

Fig. 9 Average pulse NP currents with injected $3^{\text {rd }}$ harmonic PWM method under VVVF control mode.

\section{B. Phase Pulse Width Duty Cycle Disturbance PWM Method}

In terms of (10) and (14), factors $m, I, \varphi$ etc, can be considered as disturbance sources in the capacitor-voltage loop (in Section III. C). These factors are directly associated with the $R L$ loads, and their combined effect would influence the amplitude of the synthesized average pulse NP current. Based on the referred basic carrier-based PD PWM method with saddle modulation waves, the phase pulse width duty cycle disturbance PWM method (in "Phase-a") is demonstrated in Fig. 10. When $m=0.533, I=3.4 \mathrm{~A}, \cos \varphi=0.59$ and $f=25 \mathrm{~Hz}$, the second zero sequence component $u_{\mathrm{pr}}=u_{3}$ can be obtained through the quasi PR controller, according to the capacitor voltage error $\Delta u_{12}$. Then, $u_{3}$ can be injected into the referred saddle modulation wave $u_{\text {ref_a }}^{\prime}$. Therefore, the original phase pulse width duty cycle $d_{\mathrm{a}}^{\prime}$ is modified to be $d_{\mathrm{a}}^{\prime \prime}$ according to the final modulation wave $u_{\text {ref_a }}^{\text {ea }}$, as shown in Fig. 10(a). Because the disturbance information is transferred to modulation waves by $u_{3}$ in real time, samples and calculations for phase currents and load power factors can be omitted completely.

If the VVVF operation condition is below $m=1, I=7.4 \mathrm{~A}$, $\cos \varphi=0.886$ and $f=50 \mathrm{~Hz}$, the output $u_{\mathrm{pr}}$ of the quasi PR controller (in Section III. C) may suffer from over modulation (in certain parts) according to the capacitor voltage error $\Delta u_{12}$, which is different from the case in Fig. 10(a). In order to avoid 
load current distortion, it is necessary to limit the amplitude of $u_{\mathrm{pr}}$ in terms of $\left|u_{\mathrm{pr}}+u_{\text {ref_x }}^{\prime}\right| \leq 1$ [25], where x stands for Phases a, $\mathrm{b}$, and c. It is also noted that the $\max \left(u_{\text {ref_a }}^{\prime}, u_{\text {ref_b }}^{\prime}, u_{\text {ref_c }}^{\prime}\right)$ and $\min \left(u_{\text {ref_a }}^{\prime}, u_{\text {ref_b }}^{\prime}, u_{\text {ref_c }}^{\prime}\right)$ stand for the maximum and minimum instantaneous saddle waves in every fundamental period, so $u_{\mathrm{pr}}$ can be limited as follows

$$
-1-\min \left(u_{\text {ref_a }}^{\prime}, u_{\text {ref_b }}^{\prime}, u_{\text {ref_c }}^{\prime}\right) \leq u_{\text {pr }} \leq 1-\max \left(u_{\text {ref_a }}^{\prime}, u_{\text {ref_b }}^{\prime}, u_{\text {ref_c }}^{\prime}\right)
$$

According to (15), the second zero sequence component $u_{3}$ can be obtained from $u_{\mathrm{pr}}$ as shown in Fig. 10(b). Then the original phase pulse width duty cycle $d_{\mathrm{a}}^{\prime}$ is also modified to be $d_{\mathrm{a}}^{\prime \prime}$ due to the final modulation wave $u_{\text {ref_a }}^{\prime \prime}$. Furthermore, $u_{\text {ref_a }}^{\prime \prime}$ is clamped at " 1 " to prevent over modulation and this is beneficial to reduce the switching number in each fundamental period, lowering the switching loss of the inverter.

From Fig. 10, it can be concluded that the disturbance PWM method optimizes the charging and discharging balanced states between $\mathrm{C}_{\text {cap1 }}$ and $\mathrm{C}_{\text {cap2 }}$ in terms of the asymmetric modulation wave obtained.

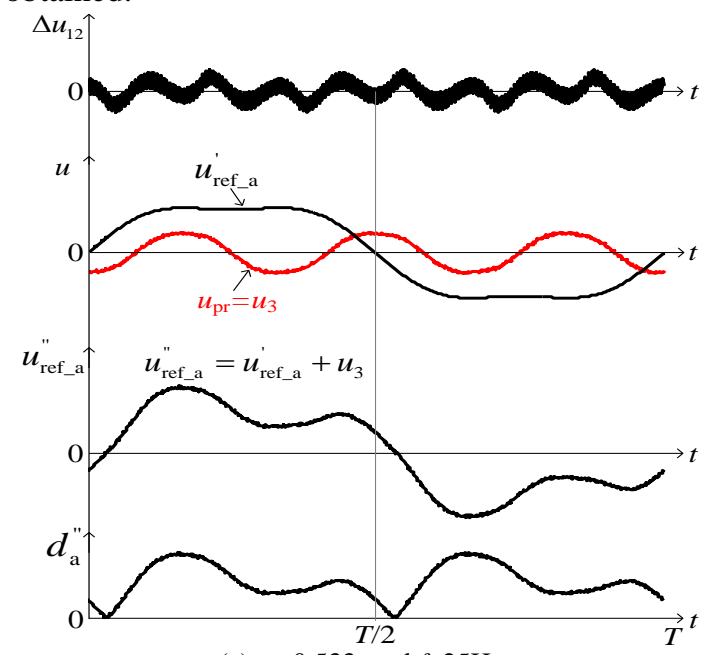

(a) $m=0.533$, and $f=25 \mathrm{~Hz}$

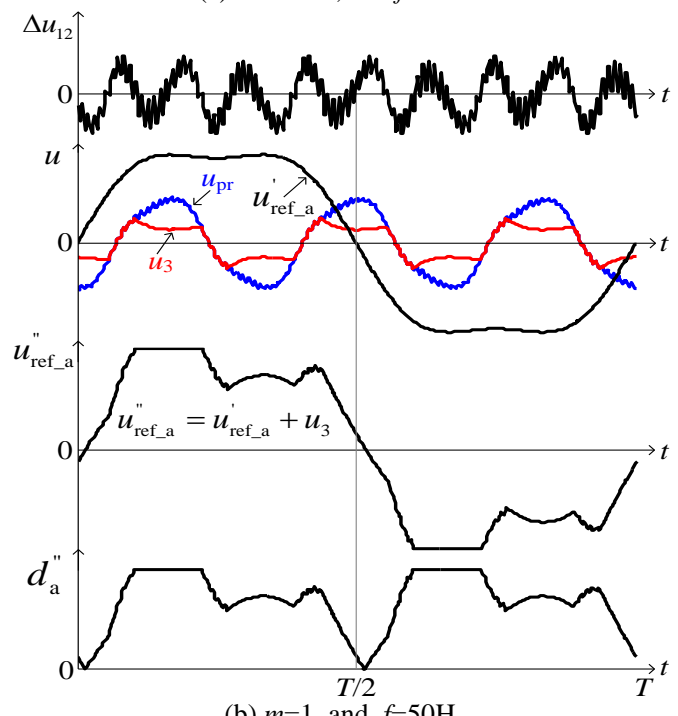

(b) $m=1$, and $f=50 \mathrm{H}$

Fig. 10 Phase pulse width duty cycle disturbance PWM method in capacitor-voltage loop (in "Phase-a").

\section{Capacitor-Voltage Loop Control with Quasi PR Controller}

If a different injected harmonic could cause the proper average pulse NP current to make the final actual synthesized average pulse NP current closer to zero, the NPP fluctuation $\Delta u_{\mathrm{o}}$ would be suppressed close to zero. In fact, the voltage difference $\Delta u_{12}=U_{\text {cap1 }}-U_{\text {cap2 }}$ directly reflects the essence of $\Delta u_{\mathrm{o}}$. In terms of the basic analysis of the NPP fluctuation in Section II $(\mathrm{A} \sim \mathrm{C}), \Delta u_{12}$ is the alternating component with the triple fundamental frequency, especially as it contains information on the load current amplitude, power factor etc. Its amplitude, frequency and equivalent initial phase change with loading.

Therefore, $\Delta u_{12}$ could be a second injected zero sequence component, which can suppress the final synthesized average pulse NP current efficiently. To achieve this, a capacitor-voltage loop control method with a quasi PR controller is proposed for the suppression of the fluctuation of the NPP as shown in Fig. 11. In terms of $U_{\text {cap } 1}+U_{\text {cap2 } 2} \equiv U_{\mathrm{dc}}$, the capacitor voltages fluctuate in opposite directions, and therefore $U_{\text {cap2 }}$ would follow $U_{\text {cap1 }}$ infinitely according to the feedback mechanism.

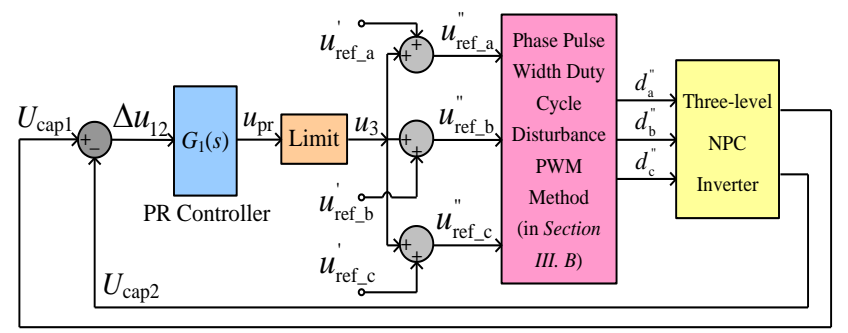

Fig. 11 Capacitor-voltage loop control method with quasi PR controller for fluctuation-suppression of NPP.

The actual second zero sequence component $u_{3}$ can be obtained after $\Delta u_{12}$ is imported into the controller. The transfer function is $G_{1}(s)$ :

$$
u_{\mathrm{pr}}=\Delta u_{12} \cdot G_{1}(s)
$$

where $\Delta u_{12}$ is also the input error to this controller, and this alternating input error can be constrained effectively by the nonlinear quasi PR controller [26]. Its transfer function is written as

$$
G_{1}(s)=G_{\mathrm{PR}}(s)=k_{\mathrm{p}}+k_{\mathrm{r}} \frac{2 \omega_{\mathrm{c}} s}{s^{2}+2 \omega_{\mathrm{c}} s+\omega_{0}^{2}}
$$

where $k_{\mathrm{p}}$ is the proportional coefficient; $k_{\mathrm{r}}$ is the resonant coefficient; $\omega_{0}$ is the resonant angular frequency; $\omega_{c}$ is the cutoff angular frequency, and $\omega_{c}<<\omega_{0}$. The controller parameters have been derived from the model parameters [26] as $k_{\mathrm{p}}=0.05, k_{\mathrm{r}}=2, \omega_{\mathrm{c}}=2 \pi \times(0.02 f)$, and $\omega_{0}=2 \pi \times(3 f)$, where $f$ is the fundamental frequency.

\section{EXPERIMENTAL VERIFICATION AND ANALYSIS}

The proposed fluctuation-suppression method of the NPP for the three-level NPC inverter is verified using the experiment platform shown in Fig. 12. The digital controller is composed of DSP (TMS320F28335) and FPGA (Cyclone EP1C6Q240C8N); 
the power switches are IGBTs (FGA40N120D) used to form the main circuit of the inverter. The DC-link voltage is supplied by two DC voltage sources in series for $U_{\mathrm{dc}}=100 \mathrm{~V}$. A three-phase adjustable resistor and inductor $(R L)$ were chosen as the load for the inverter. Voltages across DC-link capacitors are measured by voltage sensors. Phase-a modulation wave can be displayed from a DA converter $(0.175 / \mathrm{V})$. Finally, the inverter with the three-phase adjustable $R L$ load operates under VVVF control mode, and experimental parameters are listed in TABLE I.

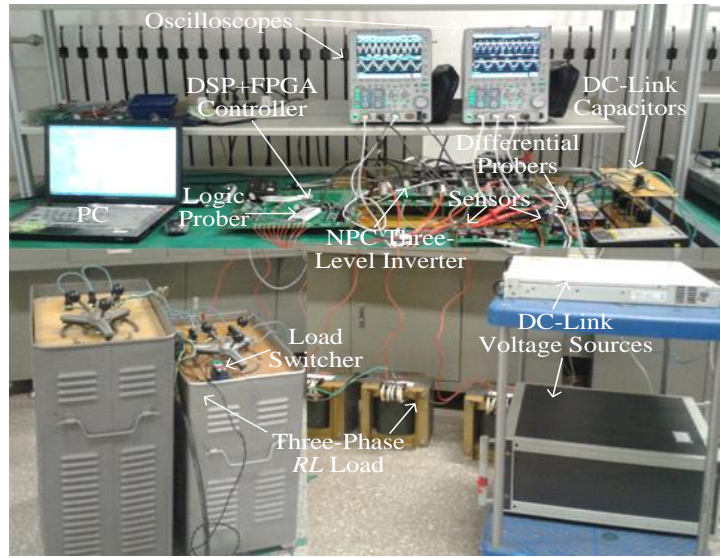

Fig. 12 Experiment platform for the three-level NPC inverter.

TABLE I Experimental parameters

\begin{tabular}{cc}
\hline \hline Parameter & Value \\
\hline DC-Link voltage $U_{\mathrm{dc}}$ & $100 \mathrm{~V}$ \\
DC-Link capacitance $C_{1}=C_{2}$ & $470 \mu \mathrm{F}$ \\
Resistor and inductor of $R L$ load & $R=0 \sim 15 \Omega, L=5 \sim 40 \mathrm{mH}$ \\
Switching frequency $f_{\mathrm{c}}$ & $4.67 \mathrm{kHz}$ \\
Variable modulation index variable & $m=1 / f=50 \mathrm{~Hz}, m=0.733 / f=40 \mathrm{~Hz}$, \\
frequency of VVVF control mode & $m=0.533 / f=25 \mathrm{~Hz}$ \\
Variable load power factor under & $0.886(m=1), 0.59(m=0.533)$ \\
VVVF control & \\
\hline \hline
\end{tabular}

The experimental results using the basic and proposed control methods are shown in Fig. 13. The amplitudes of the capacitor voltages $U_{\text {cap1 }}$ and $U_{\text {cap2 }}$ for the basic control are approximately $10 \%$ of $U_{\mathrm{dc}} / 2$ as shown in Fig. 13(a). Under the same conditions, the amplitudes of $U_{\text {cap1 }}$ and $U_{\text {cap2 }}$ decrease significantly with the proposed control as shown in Fig. 13(b). Moreover, the proposed method reduces the switching number in a fundamental period by limiting the maximum value of the final modulation wave $u_{\text {ref_a }}^{\prime \prime}$ as shown in Fig. 13(b), and there is no overlapping of level layers in the line to line voltage with both methods, comparing with the $\mathrm{NTV}^{2}$, the hybridization of the NTV and the NTV ${ }^{2}$ methods.

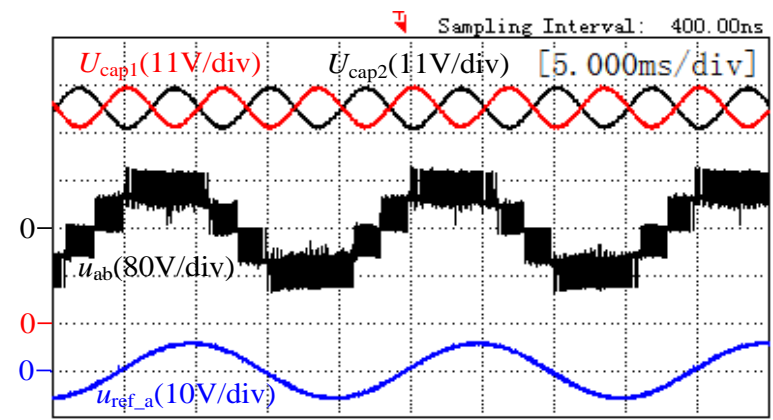

(a) Sinusoidal modulation wave (bottom), line to line voltage (middle) and capacitor voltages (upper) using basic carrier-based PD PWM method

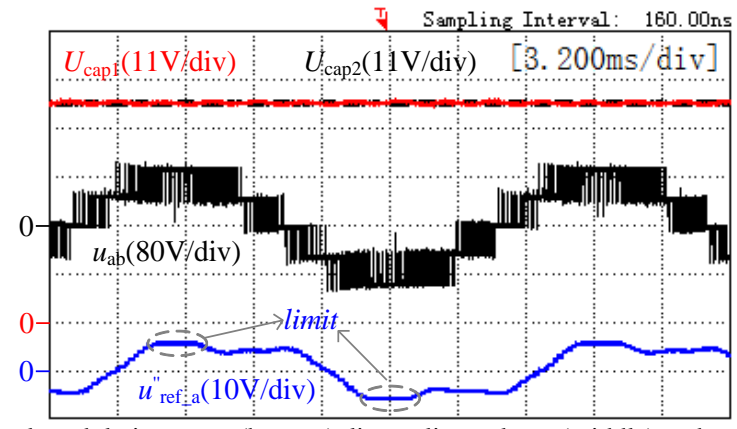

(b) Final modulation wave (bottom), line to line voltage (middle) and capacitor voltages (upper) using proposed PWM method

Fig. 13 Experimental results using basic and proposed methods $(m=1, f=50 \mathrm{~Hz}$, $\cos \varphi=0.886, R=6 \Omega, L=10 \mathrm{mH}$ and $\left.C_{1}=C_{2}=470 \mu \mathrm{F}\right)$.

When the variable modulation index, fundamental frequency and load power factor decrease following a change in operating setpoint, both $m$ and $I$ decrease. However, the fundamental period increases, and the fluctuation of $U_{\text {cap1 }}$ and $U_{\text {cap2 }}$ is still larger with the basic carrier-based PD PWM method, approximately $5 \%$ of $U_{\mathrm{dc}} / 2$ as shown in Fig. 14(a). Under the same conditions, comparing $u_{\text {ref_a }}$ with $u_{\text {ref_a }}^{\prime \prime}$ in Fig. $14(\mathrm{a}, \mathrm{b})$, the final modulation wave $u_{\text {ref_a }}^{\prime \prime}$ controlled by the proposed method is modified with the reduced modulation index, fundamental frequency and load power factor. As a result, the fluctuation of $U_{\text {cap } 1}$ and $U_{\text {cap2 }}$ is further reduced as shown in Fig. 14(b).

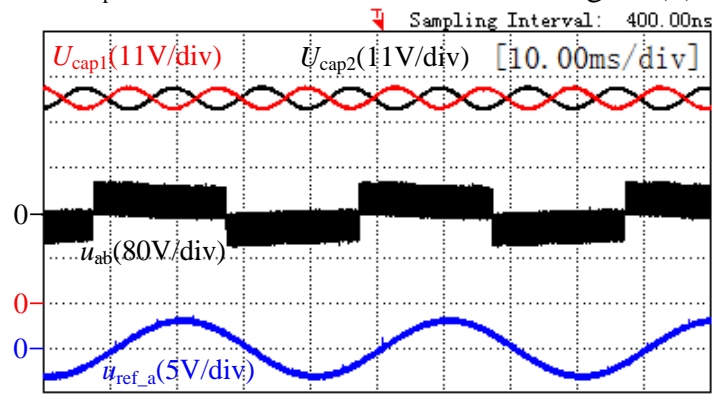

(a) Sinusoidal modulation wave (bottom), line to line voltage (middle) and capacitor voltages (upper) using basic carrier-based PD PWM method

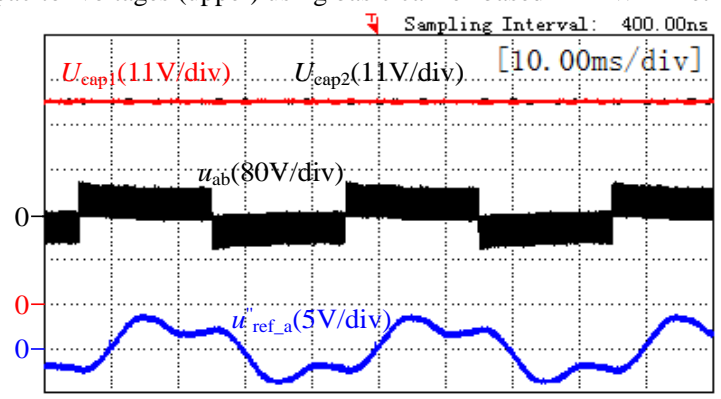

(b) Final modulation wave (bottom), line to line voltage (middle) and capacitor voltages (upper) using proposed PWM method

Fig. 14 Experimental results using basic and proposed methods $(m=0.533$, $f=25 \mathrm{~Hz}, \cos \varphi=0.59, R=4.5 \Omega, L=40 \mathrm{mH}$ and $\left.C_{1}=C_{2}=470 \mu \mathrm{F}\right)$.

In order to verify the fundamental period effect on the fluctuation, the fundamental frequency is changed from $50 \mathrm{~Hz}$ to a half $(25 \mathrm{~Hz})$, and the inductor is also changed from $10 \mathrm{mH}$ to 20mH: $m=1$ and the other parameters are kept the same as those used for Fig. 13. The phase current amplitude and the load power factor remain unchanged. As a result, with the basic carrier-based PD PWM method, $U_{\text {cap } 1}$ and $U_{\text {cap2 }}$ fluctuate by about $20 \%$ of $U_{\mathrm{dc}} / 2$ shown in Fig. 15(a), i.e. twice those in Fig. 
13 (a). It is consistent with the analytic result depicted in Fig. 4. However, the fluctuation of $U_{\text {cap1 }}$ and $U_{\text {cap2 }}$ is suppressed efficiently as shown in Fig. 15(b), to about $2 \%$ of $U_{\mathrm{dc}} / 2$ using the proposed PWM method. Therefore, the NPP fluctuation is almost eliminated by the proposed PWM method.

It is also noted that although the capacitance of the DC-link capacitors is reduced by half, and the other parameters are the same as those used in Fig. 13, the fluctuation of $U_{\text {cap1 }}$ and $U_{\text {cap2 } 2}$ is still suppressed well using the proposed PWM method as shown in Fig. 16, to about one fifth of that shown in Fig. 13(b). Thus, it is concluded that the proposed PWM method is beneficial for reducing the capacitance of the DC-link capacitors.

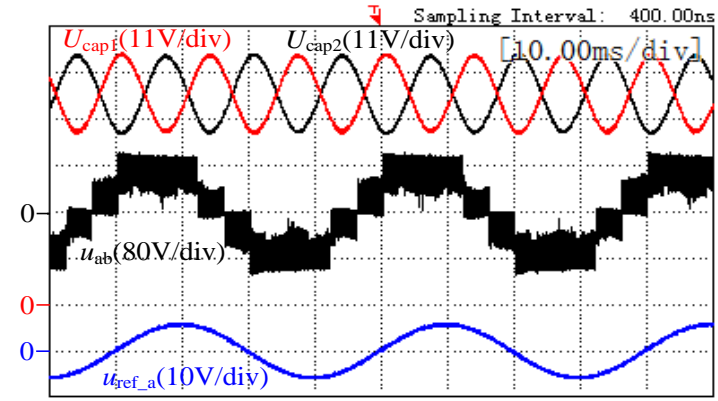

(a) Sinusoidal modulation wave (bottom), line to line voltage (middle) and capacitor voltages (upper) using basic carrier-based PD PWM method

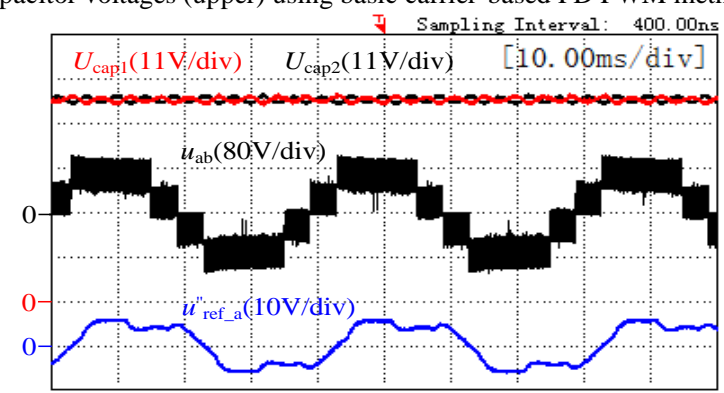

(b) Final modulation wave (bottom), line to line voltage (middle) and capacitor voltages (upper) using proposed PWM method

Fig. 15 Experimental results using basic and proposed PWM methods when fundamental frequency is reduced by half $(m=1, f=25 \mathrm{~Hz}, \cos \varphi=0.886$,

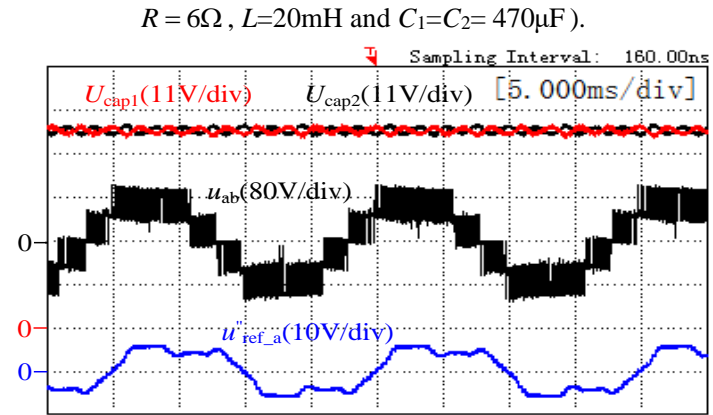

Fig. 16 Experimental results using proposed PWM method when capacitance of DC-link capacitors is reduced by half $(m=1, f=50 \mathrm{~Hz}, \cos \varphi=0.886$,

$$
R=6 \Omega, L=10 \mathrm{mH} \text { and } C_{1}=C_{2}=235 \mu \mathrm{F} \text { ). }
$$

Because conventional charge pumps may suffer from current mismatch, a $10 \%$ mismatch in one phase of the $R L$ load $\left(R_{\mathrm{b}}=R_{\mathrm{c}}=6 \Omega, L_{\mathrm{b}}=L_{\mathrm{c}}=10 \mathrm{mH}\right.$, and $\left.R_{\mathrm{a}}=6.6 \Omega, L_{\mathrm{a}}=11 \mathrm{mH}\right)$ has also been considered in this paper. Experimental results under this condition are shown using the proposed PWM method in Fig. 17. Comparing $i_{\mathrm{a}}$ with $i_{\mathrm{b}}$, (although the amplitude of $i_{\mathrm{a}}$ decreases because of $10 \%$ mismatch in Phase-a $R L$ load), the final actual synthesized average pulse NP current is still suppressed well through the capacitor-voltage loop. $U_{\text {cap1 }}$ and $U_{\text {cap2 }}$ have very little difference to those under the symmetrical three-phase $R L$ load as shown in Fig. 13(b).

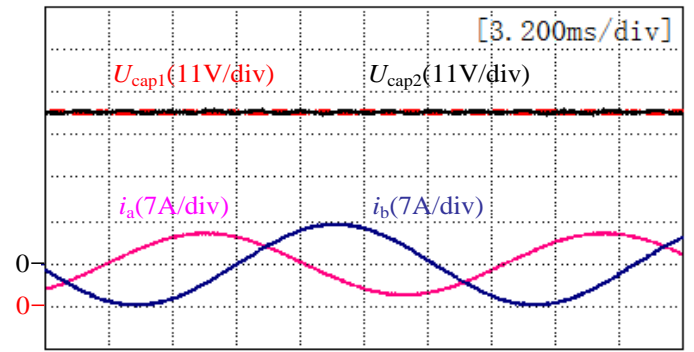

Fig. 17 Experimental results of mismatch $10 \%$ in Phase-a $R L$ load using proposed PWM method $\left(m=1, f=50 \mathrm{~Hz}, R_{\mathrm{b}}=R_{\mathrm{c}}=6 \Omega, L_{\mathrm{b}}=L_{\mathrm{c}}=10 \mathrm{mH}, R_{\mathrm{a}}=6.6 \Omega\right.$,

$$
L_{\mathrm{a}}=11 \mathrm{mH} \text {, and } C_{1}=C_{2}=470 \mu \mathrm{F} \text { ) }
$$

Another mismatch that may happen in the three-level NPC inverter is the capacitance imbalance between the DC-link capacitors, and it may result in the failure of the power switches due to the serious unbalanced voltage stress, as well as the distortion of the line to line voltages. In order to demonstrate the ability of fluctuation-suppression of the proposed PWM method, the equivalent $C_{2}$ is changed from $470 \mu \mathrm{F}$ (paralleled by two capacitors with capacitance $235 \mu \mathrm{F}$ ) to $235 \mu \mathrm{F}$ (removing one of the two capacitors). By adjusting the final modulation wave at each switching period, the capacitor voltages $U_{\text {cap1 }}$ and $U_{\text {cap2 }}$ fluctuate only a little more as shown in Fig. 18, and the line to line voltage $u_{\mathrm{ab}}$ is not influenced significantly. Therefore, the proposed method can tolerate the imbalance capacitance (at least 50\%) of the series connected DC-link capacitors, due to the capacitor-voltage loop.

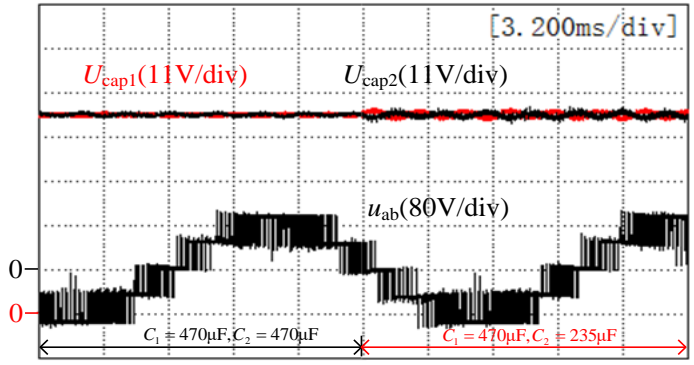

Fig. 18 Experimental results of capacitance imbalance between DC-link capacitors using proposed PWM method $(m=1, f=50 \mathrm{~Hz}, \cos \varphi=0.886$,

$R=6 \Omega, L=10 \mathrm{mH}, C_{1}=470 \mu \mathrm{F}$, and equivalent $C_{2}$ is changed from $470 \mu \mathrm{F}$ to $235 \mu \mathrm{F})$.

When a symmetric three-phase load step change happens from $R=9 \Omega$ to $4.5 \Omega$, the experiment results are shown in Fig. 19 . With the proposed method, $i_{\mathrm{a}}$ and the fluctuation of $U_{\text {cap1 }}$ and $U_{\text {cap2 }}$ increase, as shown in Fig. 19(a, b) respectively. However, the final modulation wave $u_{\text {ref_a }}^{\prime \prime}$ is modified through the capacitor-voltage loop with the quasi PR controller, and its amplitude also increases properly as shown in Fig. 19(a) and therefore the fluctuation of $U_{\text {cap } 1}$ and $U_{\text {cap2 }}$ can also be controlled well as shown in Fig. 19(b).

Under the same conditions, Fig. 19(c) shows the load step change experiment results with the basic carrier-based PD PWM method. It is seen that the fluctuation of $U_{\text {cap } 1}$ and $U_{\text {cap2 }}$ is still much larger than the one in Fig. 19(b). After the step change in load resistance, the phase current increases and the load power factor reduces. Comparing Fig. 19(b) with Fig. 19(c), the 
fluctuation using the proposed method is virtually immune to the disturbance factors, but the basic method is very oscillatory.

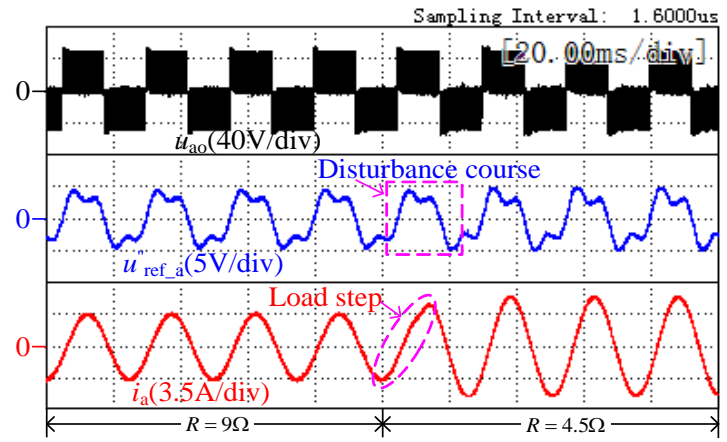

(a) Phase current (bottom), final modulation wave (middle), and phase voltage (upper) in Phase-a using proposed PWM method
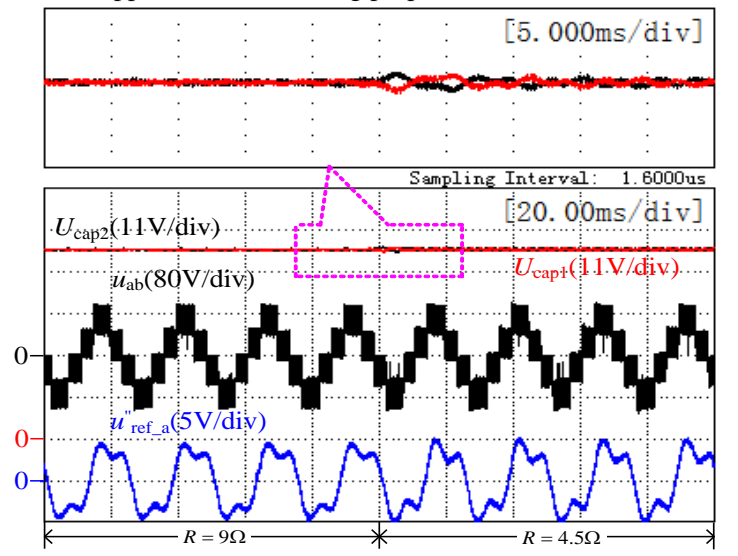

(b) Final modulation wave in Phase-a (bottom), line to line voltage (middle) and capacitor voltages (upper) using proposed PWM method
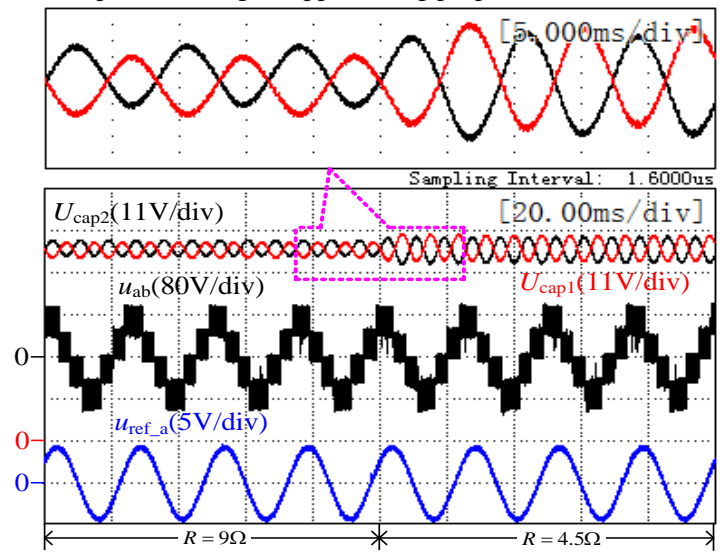

(c) Final modulation wave in Phase-a (bottom), line to line voltage (middle) and capacitor voltages (upper) using basic PWM method

Fig. 19 Experimental results of symmetric three-phase load step change from $R=9 \Omega$ to $4.5 \Omega\left(m=0.733, f=40 \mathrm{~Hz}, L=20 \mathrm{mH}\right.$ and $\left.C_{1}=C_{2}=470 \mu \mathrm{F}\right)$.

\section{Conclusion}

This paper introduces a new method for the suppression of fluctuations in the NPP of a three-level NPC inverter using the capacitor-voltage loop. Due to the constant initial phase of the injected zero sequence, the saddle wave modulation method has limited capability to suppress the fluctuation of the NPP. The essence of the NPP fluctuation can be revealed by the average pulse NP current: the fluctuation frequency is three times the fundamental; the fluctuation amplitude is mainly determined by the modulation index, the phase current and load power factor; the equivalent initial phase of the NPP fluctuation is related to the load power factor.

Reducing the amplitude of the average pulse NP current is an efficient way to suppress the NPP fluctuation. Under VVVF control mode, the controlled variable, which varies with the disturbance factors, (i.e. modulation index, phase current, load power factor, etc) can be produced properly by a quasi PR controller in the capacitor-voltage loop. The final three-phase modulation waves are obtained by virtue of the controlled variable. The reduction in amplitude of the average pulse NP current is carried out through the proposed phase pulse width duty cycle disturbance PWM. Experimental results demonstrate that under the VVVF control mode, the proposed method not only suppresses the NPP fluctuation efficiently, but also avoids the overlapping of the level layers in the line to line voltages, reduces the switching actions (in high modulation indices), and tolerates the imbalance capacitance (at least 50\%) of the series connected DC-link capacitors. It is suitable for fan and pump applications with the induction motor.

\section{REFERENCES}

[1] A. Nabae, I. Takahashi, and H. Akagi, "A new neutral-point-clamped PWM inverter," IEEE Trans. Ind. Appl., vol. IA-17, no. 5, pp. 518-523, Sep./Oct. 1981.

[2] D. W. Kang, C. S. Ma, T. J. Kim and D. S. Hyun, "Simple control strategy for balancing the DC-link voltage of neutral-point-clamped inverter at low modulation index," IEE Proceedings-Electric Power Applications, vol. 151, no. 5, pp. 569-575, Sep. 2004.

[3] U. Choi, J. Lee, and K. Lee, "New modulation strategy to balance the neutral-point voltage for three-level neutral-clamped inverter systems," IEEE Trans. Energy Convers., vol. 29, no. 1, pp. 91-100, Mar. 2014.

[4] J. Shen, S. Schröder, B. Duro, and R. Roesner, "A neutral-point balancing controller for a three-level inverter with full power-factor range and low distortion," IEEE Trans. Ind. Appl., vol. 49, no. 1, pp. 138-148, Jan./Feb. 2013.

[5] R. Sommer, A. Mertens, C. Brunotte, and G. Trauth, "Medium voltage drive system with NPC three-level inverter using IGBTs," in Proc. IEEE PWM Medium Voltage Drives Semin., May 11, 2000, pp. 1-5.

[6] P. C. Loh, F. Gao, and F. Blaabjerg, et al., "Pulse width-modulated Z-source neutral-point-clamped inverter," IEEE Trans. Ind. Appl., vol. 43, no. 5, pp. 1295-1308, Sep./Oct. 2007.

[7] J. Pou, R. Pindado, D. Boroyevich, and P. Rodriguez, "Limits of the neutral-point balance in back-to-back-connected three-level converters," IEEE Trans. Power Electron., vol. 19, no. 3, pp. 722-731, May 2004.

[8] C. L. Xia, X. Gu, T. N. Shi, and Y. Yan, "Neutral-point potential balancing of three-level inverters in direct-driven wind energy conversion system," IEEE Trans. Energy Convers., vol. 26, no. 1, pp. 18-29, Mar. 2011.

[9] M. K. Mishra, A. Joshi, and A. Ghosh, "Control schemes for equalization of capacitor voltages in neutral clamped shunt compensator," IEEE Trans. Power Del., vol. 18, no. 2, pp. 538-544, Apr. 2003.

[10] Z. Zhang, O. C. Thomsen, and M. A. E. Andersen, "Discontinuous PWM modulation strategy with circuit-level decoupling concept of three-level neutral-point-clamped (NPC) inverter", IEEE Trans. Ind. Electron., vol. 60, no. 5, pp. 1897-1906, May 2013.

[11] A. K. Gupta, and A. M. Khambadkone, "A simple space vector PWM scheme to operate a three-level NPC inverter at high modulation index including over modulation region, with neutral point balancing," IEEE Trans. Ind. Appl., vol. 43, no. 3, pp. 751-760, May/Jun. 2007.

[12] S. Das, and G. Narayanan, "Analytical closed-form expressions for harmonic distortion corresponding to novel switching sequences for neutral-point-clamped inverters", IEEE Trans. Ind. Electron., vol. 61, no. 9, pp. 4485-4497, May 2014.

[13] Y. C. Zhang, Z. M. Zhao, and J. G. Zhu, "A hybrid PWM applied to high-power three-level inverter-fed induction-motor drives", IEEE Trans. Ind. Electron., vol. 58, no. 8, pp. 3409-3420, May 2011.

[14] O. Dordevic, M. Jones, and E. Levi, "A comparison of carrier-based and space vector PWM techniques for three-level five-phase voltage source inverters", IEEE Trans. Ind. Inform., vol. 9, no. 2, pp. 609-619, May 2013. 
[15] L. Ben-Brahim, and S. Tadakuma, "A Novel multilevel carrier-based PWM-control method for GTO inverter in low index modulation region," IEEE Trans. Ind. Appl., vol. 42, no. 1, pp. 121-127, Jan./Feb. 2006.

[16] L. Masisi, Pragasen. Pillay, and S. Williamson, "An efficient control strategy for a five-level inverter comprising flying-capacitor asymmetric H-bridgeA modulation strategy for a three level inverter synchronous reluctance motor (SynRM) drive," IEEE Trans. Ind. Appl., DOI 10.1109/TIA.2015.2497307, 2015.

[17] Y. Zhang and L. Sun, "An efficient control strategy for a five-level inverter comprising flying-capacitor asymmetric H-bridge," IEEE Trans. Ind. Electron., vol. 58, no. 9, pp. 4000-4009, Sep. 2011.

[18] S. Busquets-Monge, J. Bordonau, D. Boroyevich, and S. Somavilla, "The nearest three virtual space vector PWM-A modulation for the comprehensive neutral-point balancing in the three-level NPC inverter," IEEE Power Electron. Lett., vol. 2, no. 1, pp. 11-15, Mar. 2004.

[19] S. Busquets-Monge, S. Somavilla, J. Bordonau, and D. Boroyevich, "Capacitor voltage balance for the neutral-point-clamped converter using the virtual space vector concept with optimized spectral performance," IEEE Trans. Power Electron., vol. 22, no. 4, pp. 1128-1135, Jul. 2007.

[20] C. L. Xia, H. J. Shao, Y. Zhang, and X. N. He, "Adjustable Proportional Hybrid SVPWM Strategy for Neutral-Point-Clamped Three-Level Inverters," IEEE Trans. Ind. Electron., vol. 60, no. 10, pp. 4234-4242, Oct. 2013.

[21] B. Lazhar, "A discontinuous PWM method for balancing the neutral point voltage in three-level inverter-fed variable frequency drives," IEEE Trans. Energy Convers., vol. 23, no. 4, pp. 1057-1063, Dec. 2008.

[22] A. K. Gupta, and A. M. Khambadkone, "A speed-sensorless start-up method of an induction motor driven by a modular multilevel cascade inverter (MMCI-DSCC)," IEEE Trans. Ind. Appl., vol. 50, no. 4, pp. 2671-2680, Jul./Aug. 2014.

[23] S. Payami, R. K. Behera, A. Iqbal, and R. Al-Ammari, "Common-mode voltage and vibration mitigation of a five-phase three-level NPC inverter-fed induction motor drive system," IEEE Journal of Emerging and Selected Topics in Power Electron., vol. 3, no. 2, pp. 349-361, Jun. 2015.

[24] X. Yuan, Y. Li, and C. Wang, "Objective optimization for multilevel neutral-point-clamped converters with zero-sequence signal control," IET Power Electron., vol. 3, no. 5, pp. 755-763, Sep. 2009.

[25] R. Zhu, X. Wu, and Y. Tang, "Duty cycle-based three-level space-vector pulse-width modulation with overmodulation and neutral-point balancing capabilities for three-phase neutral-point clamped inverters," IET Power Electron., vol. 8, no. 10, pp. 1931-1940, Apr. 2015.

[26] C. Xia, F. Zhou, Z. Wang, and X. He, "Equivalent switch circuit model and proportional resonant control for triple line-voltage cascaded voltage-source converter," IEEE Trans. Power Electron., vol. 28, no. 5, pp. 2389-2401, May 2013.

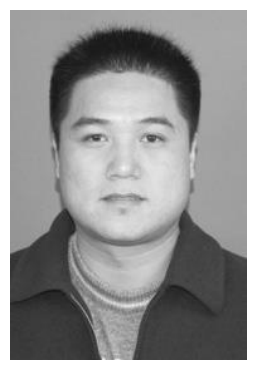

Yun Zhang (M'13) was born in Jiangsu, China, in 1980. He received the B.S. and M.S. degrees from Harbin University of Science and Technology, and the $\mathrm{Ph} . \mathrm{D}$. degree from Harbin Institute of Technology, Harbin, China, in 2003, 2006 and 2010 respectively, all in electrical engineering.

He is currently an Associate Professor in the School of Electrical Engineering and Automation, Tianjin University, Tianjin, China. His current research interests include topologies, modulation and control strategies of power converters for microgrid and

electric vehicles.

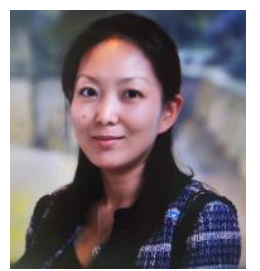

Jing Li received her MS.c (Distinction) and B.Eng. (Honour) from Beijing Institute of Technology, obtained the Ph.D degree from the University of Nottingham, UK in 2010. She subsequently worked as a research fellow within the Power Electronic, Machine and Control Group in University of Nottingham, UK.

She is currently a lecture in the Electrical and Electronic Engineering Department, University of Nottingham, Ningbo, China. Her research interests are condition monitoring for motor drive systems and power distribution systems, advanced control and design of motor drive systems.

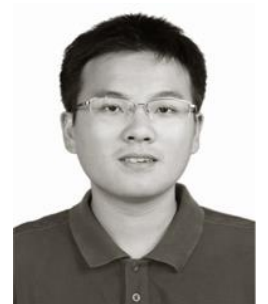

Xinmin Li was born in Hunan, China, in 1989. He received the B.S. degree from University of Science and Technology Beijing, Beijing, China, in 2011.

$\mathrm{He}$ is currently working toward the Ph.D. degree in electrical engineering in the School of Electrical Engineering and Automation, Tianjin University, Tianjin, China. His research interests include electrical machines and motor drives, power electronics and wind power technology.

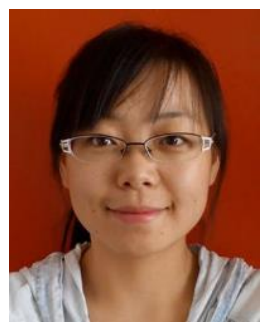

Yanfei Cao was born in Hebei, China, in 1990. She received the B.S. degree in automation from Hebei University of Technology, Tianjin, China, in 2013. She is currently working toward the Ph.D. degree in electrical engineering in the School of Electrical Engineering and Automation, Tianjin University, Tianjin, China.

Her research interests include electrical machines, motor drives and power electronics.

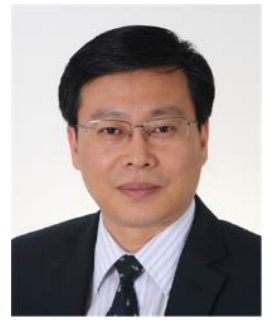

Changliang Xia (M'08-SM'12) was born in Tianjin, China, in 1968. He received the B.S. degree from Tianjin University, China, in 1990, and the M.S. and $\mathrm{Ph} . \mathrm{D}$. degrees from Zhejiang University, China, in 1993 and 1995 respectively, all in electrical engineering.

$\mathrm{He}$ is currently a Professor in the School of Electrical Engineering and Automation, Tianjin University, and also in Tianjin Key Laboratory of Advanced Technology of Electrical Engineering and Energy, Tianjin Polytechnic University. In 2008, he became "Yangtze Fund Scholar" Distinguished Professor and is currently supported by National Science Fund for Distinguished Young Scholars. His research interests include electrical machines and their control systems, power electronics, and control of wind generators. 